\begin{tabular}{|c|c|}
\hline Title & Influence of liquid-solid intermolecular force on levitation of impacting nanodroplet \\
\hline Author(s) & Tabe, Hirofumi; Kobay ashi, Kazumichi; Y aguchi, Hisao; Fujii, Hiroy uki; W atanabe, Masao \\
\hline Citation & $\begin{array}{l}\text { Heat and Mass Transfer, 55(4), 993-1003 } \\
\text { https://doi.org/10.1007/\$00231-018 2474-4 }\end{array}$ \\
\hline Issue Date & $2019-04$ \\
\hline DOC URL & http:/hdl.handle.net/2115/77197 \\
\hline Rights & The final publication is avai lable at link.springer.com. \\
\hline Type & article (author version) \\
\hline File Information & Tabe_HAMT 2018_HUSCAP.pdf \\
\hline
\end{tabular}

Instructions for use 


\title{
Influence of liquid-solid intermolecular force on levitation of impacting nanodroplet
}

\author{
Hirofumi Tabe, Kazumichi Kobayashi, \\ Hisao Yaguchi, Hiroyuki Fujii, Masao \\ Watanabe
}

Received: date / Accepted: date

\begin{abstract}
When droplets impact on a heated wall, they can levitate owing to the vapor stream generated by the droplet evaporation. This phenomenon is called the Leidenfrost effect, and the vapor layer prevents heat transfer between the droplet and heated wall. In this study, we investigated the influence of the intermolecular force between liquid and solid molecules on the levitating phenomenon, which is caused by heat transfer, for nanodroplets. We used a molecular dynamics (MD) simulation to evaluate the detailed behavior of droplet levitation and investigated the temperature field of the impacting droplet. We found that the droplet levitation was likely to occur at lower temperature when the intermolecular force was stronger. In addition, when the intermolecular force was strong enough, the liquid molecules stayed on the heated wall and an adsorption layer was formed. This adsorption layer exceeded the critical temperature of the liquid molecules, and the existence of the adsorption layer significantly affected the onset of the droplet levitation.
\end{abstract}

Keywords Molecular dynamics simulation · Leidenfrost effect · Wettability • Intermolecular force · Adsorption layer

\section{Introduction}

The impact of droplets on a heated wall can be seen in spray cooling for heated steel, electronic devices, and other settings and applications [1, 2]. The utilized droplets have become smaller (tens of a micrometer) and faster (tens of $\mathrm{m} / \mathrm{s}$ ) with the recent progression of technology [3]. When a droplet impacts

K. Kobayashi

Division of Mechanical and Space Engineering, Hokkaido University, Kita 13 Nishi 8, Kitaku, Sapporo, Hokkaido, 060-8628 Japan

Tel.: +81-11-7066429

Fax: +81-11-7066429

E-mail: kobakazu@eng.hokudai.ac.jp 
on a solid wall at sufficiently high temperature, it can levitate owing to the vapor stream generated by its evaporation in the vicinity of the wall. This phenomenon is called the Leidenfrost effect [4], and the heat transfer between the droplet and heated wall is prevented by the vapor layer, which has a low thermal conductivity. This effect is also applied in the field of microfluidics $[5,6]$.

The boiling regimes of the impacting droplet on the heated wall have been extensively investigated. It is known that the behavior of the impacting droplet on the heated wall and heat transfer are influenced by both wall temperature and Weber number of the drople [7]. The Weber number, which is the ratio of the inertia force to surface tension force of the droplet, is the following dimensionless number:

$$
W e=\frac{\rho D V^{2}}{\gamma},
$$

where $\rho$ is the density of the liquid, $D$ is the diameter of the droplet, $V$ is the impact velocity, and $\gamma$ is the surface tension of the liquid, respectively. Many studies have been conducted to investigate the effect of the Weber number on droplet boiling and the onset of the Leidenfrost effect. In these studies, it was reported that the high Weber number caused by the high droplet velocity or the low surface tension increased the Leidenfrost temperature, which is the minimum temperature for the onset of the Leidenfrost effect $[8,9,10,11]$. This means that the high Weber number of a droplet suppresses the onset of the Leidenfrost effect.

In addition to the Weber number, the wettability of the solid surface also affects the behavior of the impacting droplet and droplet boiling. The wettability of the solid surface can be changed by changing the surface roughness and surface properties. In particular, the influence of textures on the Leidenfrost temperature has been widely investigated. Some studies showed that the existence of the textures on the solid surface increased the Leidenfrost temperature $[12,13]$, while others indicated opposite results $[10,14]$. Clavijo et al. [15] investigated the Leidenfrost temperature with hydrophilic and hydrophobic walls of plane surfaces, and superhydrophilic and superhydrophobic walls of textured surfaces. In their study, although the Leidenfrost temperature became high as the solid surface became hydrophilic from superhydrophobic, the Leidenfrost temperature of the superhydrophilic surface was lower than that of the hydrophilic surface. Furthermore, Kwon et al. [16] reported that the dense textures of a hydrophilic surface decreased the Leidenfrost temperature because the outward vapor flow was hindered by these dense textures, while the sparse textures of a hydrophilic surface raised the Leidenfrost temperature.

Many studies have been conducted to investigate the influence of the wettability on the Leidenfrost temperature. However, the dependence of the Leidenfrost temperature on the wettability of a solid surface is not well understood because there are numerous factors involved in the wettability, as mentioned above. Therefore, detailed analyses on the mechanisms or conditions for the onset of the droplet levitation from the viewpoint of wettability, which have not been clarified yet, are needed. 
In this study, we investigated the influence of the wettability of a solid surface on the droplet levitation and the evaporation process of the impacting nanodroplets by changing the liquid-solid intermolecular force with molecular dynamics (MD) simulations. Because the MD simulation can treat phase-change phenomena without any specific model, we can analyze the evaporation process, which is essentially a molecular scale and non-equilibrium phenomenon. For instance, the evaporation of a sessile nanodroplet has been studied by MD simulation [17, 18].

As a further advantage of using MD simulations, we can easily change the intermolecular force between molecules. The main factors on the wettability of a solid surface are the surface roughness and intermolecular force between liquid and solid molecules. Previous researches have shown that the wettability of a solid surface was changed by varying the intermolecular force between liquid and solid molecules in the MD simulation [19, 20, 21]. In general, the solid surface becomes strong hydrophilic when the liquid-solid intermolecular force becomes strong.

Because the intermolecular force is a more fundamental factor of determining the wettability than surface structures, we investigated the evaporating phenomena of the impacting nanodroplets on a flat surface by changing the liquid-solid intermolecular force. To investigate the influence of $W e$, the initial velocity of the droplet was also changed. In addition, we calculated the density and temperature fields of the impacting and evaporating droplet to analyze the detailed mechanism of the evaporating process.

\section{Method}

\subsection{System condition}

In this study, we used argon for the liquid and vapor molecules (21030 and 5740 molecules, respectively), and platinum for the solid molecules (140400 molecules). The initial diameter of the nanodroplet was $D_{0}=12.60 \mathrm{~nm}$. The initial configuration of this simulation is shown in Fig. 1. The lengths of the simulation domain were $43.3 \mathrm{~nm} \times 43.2 \mathrm{~nm} \times 40.0 \mathrm{~nm}$. Periodic boundary conditions were imposed in the $x$ and $y$ directions, and a mirror boundary condition was imposed on the top surface in the $z$ direction.

The droplet and vapor were formed in the vapor-liquid equilibrium state at $85 \mathrm{~K}[22]$. In this process, the velocity scaling method was applied to argon molecules to keep at $85 \mathrm{~K}$ in the calculation domain. In another system, the platinum molecules were arranged in three layers to be the fcc crystal with (111) surface. Under these three layers, other two layers of platinum were inserted to control the wall temperature with the Langevin method [23]. An initial wall temperature was set as $85 \mathrm{~K}$. By inserting this platinum wall to the bottom of the calculation domain, the initial configuration of this MD simulation as seen in Fig. 1 was established. 
An initial velocity of $V_{0}$ was given to the droplet molecules to impact on the heated wall. The initial velocities $V_{0}$ were changed to 100, 200, and 300 $\mathrm{m} / \mathrm{s}$. The corresponding Weber numbers were 13.6, 54.3, and 122, respectively. In this study, we carried out the high-velocity droplet impact to fit the Weber number with the previous experiments $[10,13,15,24]$ as much as possible. The applied parameters of surface tension and liquid density were $\gamma=0.013 \mathrm{~N} / \mathrm{m}$ and $\rho=1400 \mathrm{~kg} / \mathrm{m}^{3}[22]$.

After the initial velocity was given to the droplet molecules, the wall temperature was raised to the desired temperature for each simulation. Figure 2 shows examples of the time evolutions of the wall temperature. The dashed line represents the time the droplet impacts with $V_{0}=300 \mathrm{~m} / \mathrm{s}$. The wall temperature was raised sufficiently until impact, and was kept at the desired temperature through the simulation.

\subsection{Calculation model}

We used the Lennard-Jones potential function for the intermolecular force between argon molecules,

$$
\phi_{\mathrm{Ar}}(r)=4 \varepsilon_{\mathrm{Ar}}\left[\left(\frac{\sigma_{\mathrm{Ar}}}{r}\right)^{12}-\left(\frac{\sigma_{\mathrm{Ar}}}{r}\right)^{6}\right],
$$

where the potential depth and the molecule diameter are $\varepsilon_{\mathrm{Ar}}=1.635 \times$ $10^{-21} \mathrm{~J}$ and $\sigma_{\mathrm{Ar}}=0.3405 \mathrm{~nm}$, respectively. $r$ represents the distance between molecules.

The interaction between platinum molecules was represented by the following harmonic oscillator potential,

$$
\phi_{\mathrm{Pt}}(r)=\frac{1}{2} k\left(r-r_{0}\right)^{2},
$$

where $k$ is the spring constant $(k=46.8 \mathrm{~N} / \mathrm{m})$ and $r_{0}$ is the lattice constant of platinum $\left(r_{0}=0.2774 \mathrm{~nm}\right)$.

For the intermolecular force between argon and platinum molecules, we used the following potential function,

$$
\phi_{\text {int }}(r)=4 \varepsilon_{\text {int }}\left[\left(\frac{\sigma_{\text {int }}}{r}\right)^{12}-\beta\left(\frac{\sigma_{\text {int }}}{r}\right)^{6}\right],
$$

with

$$
\sigma_{\mathrm{int}}=\frac{\sigma_{\mathrm{Ar}}+\sigma_{\mathrm{Pt}}}{2}, \varepsilon_{\mathrm{int}}=\alpha \sqrt{\varepsilon_{\mathrm{Ar}} \cdot \varepsilon_{\mathrm{Pt}}},
$$

where $\varepsilon_{\mathrm{Pt}}=8.346 \times 10^{-20} \mathrm{~J}$ and $\sigma_{\mathrm{Pt}}=0.2475 \mathrm{~nm}$, respectively. From Eqs.(4) and (5), the intermolecular force between argon and platinum molecules can be changed with variations in $\alpha$ or $\beta$. The wettability of the solid surface can be changed by varying the liquid-solid intermolecular force. In this study, we fixed the value of $\beta$ as $\beta=1.0$, and changed the value of $\alpha$ for $\alpha=0.07,0.14$, and 1.0. The intermolecular force became strong as the value of $\alpha$ became high. 
MD studies, which changed wettability by varying a liquid-solid interaction parameter, have been conducted to compare behaviors of impacting droplet with experiments on several wettabilities [25, 26]

Owing to the intermolecular force between argon and platinum molecules, vapor argon molecules could adhere to the platinum wall and a thin argon film was formed on the wall before the impact of liquid droplet. This formation of the thin film occurred only the case at $V_{0}=100 \mathrm{~m} / \mathrm{s}$ because of the impact time of the droplet in this simulation. It was inconvenient that the difference in the formation occurred depending on the initial velocity of the droplet even with the same value of $\alpha$. It is difficult to establish the thin film into the initial condition beforehand with adjusting the number of the droplet and vapor molecules in consideration of the formation of the thin film. A difference in the saturated vapor pressure between the droplet and thin film also makes it difficult. To resolve these issues, we set $\alpha=0.007$ for vapor-solid intermolecular force at initial condition for all cases of this study to avoid adsorption and keep the solid surface clean before impact. In a previous MD study [25], it is reported that a droplet behavior for $\alpha=0.007$ had a good similarity with that of experiments on a superhydrophobic surface. We can say that this value is reasonable to avoid the adsorption of vapor molecules.

The time step was $5 \mathrm{fs}$, and data of every $2 \mathrm{ps}$, which were calculated by averaging 400 steps, were used for the analysis. The Newton's equation for each molecule was solved by using the leap-frog method, and a cutoff radius was set as $R_{c}=1.5 \mathrm{~nm}$.

\subsection{Wettability and liquid-solid intermolecular force}

A contact angle $\theta$ of the droplet can be calculated from the following Young's equation,

$$
\gamma_{S V}=\gamma_{L V} \cos \theta+\gamma_{S L},
$$

where $\gamma_{S V}$ is a solid-vapor interfacial tension, $\gamma_{L V}$ is a liquid-vapor interfacial tension, and $\gamma_{S L}$ is a solid-liquid interfacial tension, respectively.

Nishida et al. [21] calculated the interfacial tension balance in a hemicylindrical droplet system with several liquid-solid intermolecular forces, and obtained the contact angle from the Young's equation in Eq.(6) in a nanoscale MD simulation. They reported that the contact angle derived from the Young's equation almost corresponded to the contact angle measured from a least squares circle fitting. Therefore, the contact angle and wettability of the solid surface can be evaluated without calculating the Young's equation in a similar MD study.

Figure 3 shows the droplet shapes after reaching equilibrium from the impact on the solid wall with the initial velocity of $V_{0}=3 \mathrm{~m} / \mathrm{s}$ in this MD system. By using the circle fitting, the contact angles of droplets were measured as $\theta=70^{\circ}$ for $\alpha=0.07$, and $\theta=40^{\circ}$ for $\alpha=1.0$, respectively. This indicates that the solid wall becomes strong hydrophilic as the liquid-solid intermolecular force becomes strong. 
Some chemical approaches are used to change the wettability by varying liquid-solid intermolecular interaction without any change of surface structures. For instance, Teflon coating to yield a hydrophobic surface or plasma coating to yield hydrophilic surface is used. We can reproduce several wettabilities correlated to these applications by varying the value of $\alpha$.

\subsection{Definition of Leidenfrost effect}

In this study, we used the double-cylinder control volumes [26] shown in Fig. 4 to calculate the density or temperature field under the assumption that the droplet impact had axial symmetry. The height $d z$ and thickness $d r$ of each control volume were set as the molecule diameter of argon $\left(\sigma_{\mathrm{Ar}}\right)$, and the number of control volumes was $63 \times 63$. We also defined the Layers (Layer 1 , Layer 2, etc.) from the bottom of the control volumes, as shown in Fig. 4.

Figure 5a shows an example of the density field calculated with the control volumes. From this figure, we cannot clearly distinguish between the liquid and vapor phases, and this makes it difficult to evaluate the droplet levitation with precision. Therefore, we set a threshold density for binarization and investigated whether the droplet levitated or not by using the binary image, as shown in Fig. 5b. The threshold density for binarization was set as $540 \mathrm{~kg} / \mathrm{m}^{3}$, derived from the critical density of argon $\left(\rho_{c}=535.6 \mathrm{~kg} / \mathrm{m}^{3}\right)$.

Kobayashi et al. [26] showed that there was little influence of the intermolecular force from the wall on the liquid molecules in the fourth or higher layer of the control volumes because of the balance between the cutoff radius and height of the layer. Hence, we defined the onset of the Leidenfrost effect as the case in which the droplet (liquid phase) levitates over the Layer 4 of the control volumes. Note that, in this study, we referred to both levitation and bounce as the Leidenfrost effect on this definition.

\section{Results}

\subsection{Levitation of droplet}

Figure 6 shows the droplet shapes with $V_{0}=100 \mathrm{~m} / \mathrm{s}$ until the Leidenfrost effect occurred $\left(T_{w}=340 \mathrm{~K}\right)$ for (a) $\alpha=0.07$, (b) $\alpha=0.14$, and (c) $\alpha=1.0$, respectively. The cases $T_{w}=340 \mathrm{~K}$ were suitable for detailed analyses as the droplets can levitate regardless of other conditions. The cases $V_{0}=100 \mathrm{~m} / \mathrm{s}$ were also suitable as the droplets kept its spherical shape after impacting and levitating.

For $\alpha=1.0$, the adsorption layer was formed on the heated wall, while a small adsorption layer was formed during droplet evaporation for $\alpha=0.14$. A remaining fragment of the adsorption layer was observed at the center of the wall in Fig. 6b. And for $\alpha=0.07$, no adsorption layer was observed on the heated wall. The adsorption layer was formed when the liquid-solid intermolecular force became sufficiently strong. Figure 7 shows the intermolecular 
potential between liquid and solid molecules for each value of $\alpha$. The dashed lines represent the layer boundaries of the control volumes. For $\alpha=1.0$, a strong attractive force covers a range from Layer 1 to Layer 2. Thus, in this study, the adsorption layer was formed mainly in Layer 1 and Layer 2. Additionally, although the onset time of the Leidenfrost effect was faster, the volume of levitating droplets appeared to be smaller when the value of $\alpha$ was higher, as can be seen in Fig. 6 .

Here, we showed the influence of $\alpha$ only on the droplet shapes. However, various conditions (e.g., wall temperature, impact velocity, and liquid-solid intermolecular force) affect the onset of the Leidenfrost effect, and the droplet behavior or evaporation form of the droplet changed according to these conditions. Therefore, a detailed research on the evaporation or levitation of the droplet is needed to clarify the mechanism of the onset of the Leidenfrost effect.

\subsection{Droplet position}

We investigated the levitating process of the droplet because, in this study, we defined the Leidenfrost effect as the levitation of the droplet. We calculated the distance between the solid surface and the position of the center of gravity of the droplet $\left(z_{g}\right)$ to investigate the levitation process of the droplet with $V_{0}=100 \mathrm{~m} / \mathrm{s}$. Because the mass of each argon molecule was constant, $z_{g}$ was calculated only from the $z$ coordinates of the droplet molecules, as in the following equation:

$$
z_{g}=\frac{1}{N} \sum_{i}^{N} z^{i}
$$

where $z^{i}$ is the $z$ coordinate of molecule $i$, and $N$ is the number of molecules in the control volumes exceeding the threshold density $\left(\rho>540 \mathrm{~kg} / \mathrm{m}^{3}\right)$.

Figure 8 shows the time evolutions of $z_{g}$ of the droplet with $V_{0}=100 \mathrm{~m} / \mathrm{s}$ for (a) $\alpha=0.07$, (b) $\alpha=0.14$, and (c) $\alpha=1.0$, respectively. The abscissa is the time, and the ordinate is $z_{g}$. In Fig. 8a the time evolution is shown with four temperatures from $255 \mathrm{~K}$ to $340 \mathrm{~K}$, although the Leidenfrost effect did not occur at $255 \mathrm{~K}$. In Figs. $8 \mathrm{~b}$ and $8 \mathrm{c}$, the time evolution is shown from a minimum temperature, at which the Leidenfrost effect occurred, to $340 \mathrm{~K}$. $t=0 \mathrm{ps}$ was set at the time when the droplet impacted on the solid wall. The open circles represent the time when the Leidenfrost effect occurred. A sharp increase in $z_{g}$ occurred for $\alpha=1.0$, as shown in Fig. 8c. After the onset of the Leidenfrost effect, $z_{g}$ was calculated without the molecules of the adsorption layer. Thus, these sharp increases are caused by a separation of the liquid droplet from the adsorption layer.

For all cases $(\alpha=0.07,0.14$, and 1.0$)$, the rise of $z_{g}$ and the onset of the Leidenfrost effect occurred earlier as the wall temperature was higher. This indicates that high wall temperature led to intensive evaporation of the 
droplet in the vicinity of the wall, and the droplet might receive a strong upward force to levitate.

Moreover, the levitating speed of the droplet (slope on the graph) became constant because it acquired uniform motion after detaching from the wall. In essence, the levitating speed became faster as the wall temperature became higher, but the speeds at $T_{w}=255$ and $340 \mathrm{~K}$ do not have this tendency as shown in Figs. 8b and 8c. The mass of the levitating droplet or evaporation amount may affect the levitating speed.

\subsection{Effective radius and the number of droplet molecules}

To elucidate the influence of the wall temperature on droplet evaporation, we investigated the effective radius of the impacting droplets and the number of droplet molecules. The effective radius relates to the droplet volume, and the number of droplet molecules relates to the mass of the droplet. The effective radius was calculated from a droplet volume derived from the control volume as in the following equation,

$$
R_{e f f}=\left(\frac{3}{4 \pi} \sum_{i} \pi R_{i}^{2} d z\right)^{1 / 3}
$$

where $R_{i}$ is the droplet radius of Layer $i$ of the control volumes and $d z\left(=\sigma_{\mathrm{Ar}}\right)$ is the height of the layer of the control volumes. $R_{i}$ is calculated from the number of control volumes exceeding the threshold density $\left(\rho>540 \mathrm{~kg} / \mathrm{m}^{3}\right)$ for each layer.

The time evolution of the effective radius of the droplet and the number of argon molecules in the droplet with $V_{0}=100 \mathrm{~m} / \mathrm{s}$ are shown in Fig. 9 . The graphs in the left column are the time evolution of the effective radius, and the graphs in the right column are the time evolution of the number of argon molecules for (a) $\alpha=0.07$, (b) $\alpha=0.14$, and (c) $\alpha=1.0$, respectively. The open circles represent the time when the Leidenfrost effect occurred, and $t=0$ ps was set at the time when the droplet impacted on the solid wall. The wall temperature for each value of $\alpha$ in Fig. 9 was the same as that in Fig. 8.

At first (for $t<100 \mathrm{ps}$ ), the effective radius temporarily increased after the impact, as shown in Fig. 9 for all cases. This tendency was not observed at $T_{w}=85 \mathrm{~K}$ (isothermal with the droplet). Thus, this increase was not caused by the impact of the droplet, and we considered that this increase occurred by an expansion of the droplet caused by the increase in droplet temperature. However, in all cases, the number of molecules of the droplet decreased owing to the droplet evaporation.

Then, after the droplet expansion, falls of the effective radius caused by evaporation were observed. Especially in Fig. 9c, sharp falls occurred. This is caused by the separation of the droplet from the adsorption layer, and it is the same reason for $z_{g}$ in Fig. 8c. 
High wall temperature leads to early onset of the Leidenfrost effect, as seen in both Figs. 8 and 9. Thus, although the decrease in the effective radius was faster as the wall temperature was higher, the droplet eventually kept a larger effective radius. An earlier onset of the Leidenfrost effect led to a smaller total amount of evaporation, and larger effective radius and number of molecules were kept after levitating in the high wall-temperature cases.

From the number of molecules of the droplet, the droplet mass was evaluated. On the one hand, high wall temperature leads to intensive evaporation of the droplet, but, on the other hand, high wall temperature leads to large mass of the levitating droplet. Therefore, we conclude that the levitating speed at $T_{w}=255$ and $340 \mathrm{~K}$ in Figs. $8 \mathrm{~b}$ and $8 \mathrm{c}$ cannot be evaluated only from wall temperature.

\subsection{Leidenfrost temperature}

We showed the effects of wall temperature, intermolecular force, and impact velocity on evaporation and levitation of the droplet. Phase diagrams of the Leidenfrost effect, which include these factors, are shown in Fig. 10. The abscissa is the impact velocity of the droplet, and the ordinate is the wall temperature. The open red circle represents the case where the Leidenfrost effect occurred, the black cross represents the case in which the Leidenfrost effect did not occur, and the open blue triangle represents the case where it is difficult to distinguish the onset. To make the phase diagrams, we performed 185 different calculations by changing the value of $\alpha$, droplet velocity, and wall temperature.

The boundary temperature where the Leidenfrost effect occurred indicates the Leidenfrost temperature. At each range of impact velocity, the Leidenfrost temperature in Fig. 10a is the highest, and in Fig. 10c is the lowest of the three, i.e., the Leidenfrost temperature becomes lower as the value of $\alpha$ becomes higher. A high value of $\alpha$ indicates strong liquid-solid intermolecular force and strong hydrophilicity of the solid surface. We can conclude that the strong liquid-solid intermolecular force decreased the Leidenfrost temperature.

The difference caused by changing the impact velocity was mainly in the droplet shape after impact. The droplet became flatten after the impact as the impact velocity became high. Examples of the three patterns of phase diagram with $V_{0}=300 \mathrm{~m} / \mathrm{s}$ are shown in Fig 11 . When the initial velocity of the droplet was $300 \mathrm{~m} / \mathrm{s}$, the thin droplet tended to levitate or evaporate in fragments, as seen in Fig. 11.

The Weber number corresponding to $V_{0}=300 \mathrm{~m} / \mathrm{s}$ is 122 in this study. As shown in previous studies $[9,24]$, the high Weber number generally increases the Leidenfrost temperature due to a balance between the vapor pressure (related to the wall temperature) and inertial pressure (related to the impact velocity) of the droplet. Experimental results of previous studies [9, 10, 11, 13, 15, 24] are well consistent with this tendency. In this MD study, the droplet became flatten and the Leidenfrost temperature increased with high impact 
velocity as seen in Figs. 10 and 11. Thus, we can say that the relation between the Weber number and Leidenfrost temperature shows same tendency in experiments and nanoscale MD simulations.

In particular, for $\alpha=0.07$, the Leidenfrost effect hardly occur also at a low impact velocity of the droplet. As shown in Fig. 10, the Leidenfrost effect occurred at about $100 \mathrm{~K}$ higher than in the cases with $\alpha=0.14$ and 1.0 at $V_{0}=$ $100 \mathrm{~m} / \mathrm{s}$. The droplet kept relatively a spherical shape after impacting when the initial velocity was $100 \mathrm{~m} / \mathrm{s}$. Less spread, caused by both weak intermolecular force and low impact velocity, led to an insufficient heat transfer between the liquid droplet and heated wall. Consequently, the droplet gradually evaporated on the heated wall and, in that case, the onset of the Leidenfrost effect was suppressed.

The critical temperature of argon is $T_{c}=150.687 \mathrm{~K}$, and Fig. 10 shows that the Leidenfrost temperatures exceed $T_{c}$. Previous experiments have shown that the Leidenfrost temperature seldom exceeds a critical temperature of a using liquid $[11,15,16]$. In this study, we used the platinum wall, which had a fairly flat surface. In reality, creating such a flat surface for experiments is quite difficult. It was reported that the evaporation rate increased with increase in a contact area between liquid and rough solid wall [27, 28], and therefore, evaporation rate did not increase with the flat platinum wall. Furthermore, we used perfectly pure argon for the liquid droplet, which means that a heterogeneous nucleation seldom occurs [29]. We think that, in this study, the evaporation and boiling are harder to occur than in experiments, and the Leidenfrost temperature increases to above $T_{c}$.

In terms of the critical temperature, the tendency was different between the previous experiments and this MD study as mentioned above. However, the results of this study that the Leidenfrost temperature of impacting droplet became lower as the solid surface changed from hydrophilic $(\alpha=0.07)$ to strong hydrophilic $(\alpha=1.0)$ are well consistent with the previous experiment [15]. In oder to clarify the reason that the Leidenfrost temperature decreases as the liquid-solid intermolecular force becomes stronger, it is needed to investigate how the liquid-solid intermolecular force affects the evaporation of the impacting droplet in this MD study.

\subsection{Development of liquid-phase temperature}

For clarifying the specific influence of the intermolecular force on droplet evaporation, we calculated the temperature fields of the liquid phase. The droplet shapes and temperature fields of a droplet impacting with $V_{0}=100 \mathrm{~m} / \mathrm{s}$ on a heated wall at $T_{w}=340 \mathrm{~K}$ are shown in the three rows of Fig. 12 for (a) $\alpha=0.07$, (b) $\alpha=0.14$, and (c) $\alpha=1.0$, respectively. The left part is the droplet shape, and the right part is the temperature field for each image. The average temperatures for the layers of the control volumes corresponding to those images are shown at the bottom of Fig. 12. The black dashed line represents the critical temperature of argon $\left(T_{c}=150.687 \mathrm{~K}\right)$, and the red dashed 
line represents the wall temperature $\left(T_{w}=340 \mathrm{~K}\right)$. The four times indicate (i) the impacting time, (ii) 20 ps later from the impacting time, (iii) the time when $z_{g}$ was minimum, and (iv) the time just before the droplet detached from the wall, respectively.

The temperature of each control volume was calculated from the following equation,

$$
T=\frac{2}{3 N k_{B}} \sum_{i}^{N} \frac{1}{2} m\left(v_{t h}^{i}\right)^{2},
$$

where $k_{B}$ is the Boltzmann constant, $m$ is the mass of the argon molecule, and $v_{t h}^{i}$ is the thermal velocity of the molecule $i$. The thermal velocity $v_{t h}^{i}$ is the difference between the molecule velocity and the average velocity of the molecules in a control volume. The temperature was calculated only from the translation energy because argon is a monoatomic molecule.

As seen from the temperature fields in Fig. 12, the droplet temperature gradually increased in the vicinity of the heated wall. For $\alpha=1.0$, the adsorption layer was formed and the temperature of the adsorption layer approached the wall temperature. For $\alpha=0.14$, a little adsorption layer with lower temperature than the case for $\alpha=1.0$ was formed only during evaporation of the droplet. An adsorption layer with high temperature was stably formed as the intermolecular force was sufficiently strong.

For $\alpha=0.07$, the average temperature did not exceed $T_{c}$, while the temperature of the adsorption layer significantly exceeded $T_{c}$ from the impacting time and was almost equal to the wall temperature $T_{w}=340 \mathrm{~K}$ in Layer 1 for $\alpha=1.0$. This reveals that a strong intermolecular force leads to efficient heat transfer from the heated wall to the liquid droplet by the adsorption layer.

In conclusion, when the intermolecular force is sufficiently strong, the adsorption layer of the same temperature as the heated wall promotes heat transfer between the droplet and heated wall. Therefore, the Leidenfrost effect is likely to occur and the Leidenfrost temperature decreases as the intermolecular force becomes strong.

\section{Conclusion}

In this study, we investigated the influence of the liquid-solid intermolecular force on the Leidenfrost temperature for nanodroplets by using an MD simulation. We found that the strong liquid-solid intermolecular force decreased the Leidenfrost temperature by forming an adsorption layer on the heated wall. This adsorption layer, which was formed by a strong attractive force, exceeded the critical temperature of the liquid molecule and the temperature of this adsorption layer was almost same as that of heated wall. The adsorption layer promoted heat transfer between the heated wall and liquid droplet. Therefore, we conclude that the nanodroplet received enough heat from heated wall via adsorption layer, and the Leidenfrost temperature became lower as the liquid-solid intermolecular force became stronger. 
Acknowledgments

We would like to thank Mr. Kazuki Konno and Mr. Syohei Furuya for their contribution to this study.

\section{Compliance with ethical standards}

Conflict of interest: The authors declare that they have no conflict of interest.

\section{References}

1. Gangtao Liang and Issam Mudawar. Review of spray cooling-part 1: Single-phase and nucleate boiling regimes, and critical heat flux. International Journal of Heat and Mass Transfer, 115:1174-1205, 2017.

2. Gangtao Liang and Issam Mudawar. Review of spray cooling-part 2: High temperature boiling regimes and quenching applications. International Journal of Heat and Mass Transfer, 115:1206-1222, 2017.

3. Navid Z Mehdizadeh and Sanjeev Chandra. Boiling during high-velocity impact of water droplets on a hot stainless steel surface. In Proceedings of the Royal Society of London A: Mathematical, Physical and Engineering Sciences, volume 462, pages 3115-3131. The Royal Society, 2006.

4. David Quéré. Leidenfrost dynamics. Annual Review of Fluid Mechanics, 45:197-215, 2013.

5. Jeong Tae Ok, Eugene Lopez-Ona, Dimitris E Nikitopoulos, Harris Wong, and Sunggook Park. Propulsion of droplets on micro-and sub-micron ratchet surfaces in the leidenfrost temperature regime. Microfluidics and Nanofluidics, 10(5):1045-1054, 2011.

6. Corey Kruse, Isra Somanas, Troy Anderson, Chris Wilson, Craig Zuhlke, Dennis Alexander, George Gogos, and Sidy Ndao. Self-propelled droplets on heated surfaces with angled self-assembled micro/nanostructures. $M i$ crofluidics and Nanofluidics, 18(5-6):1417-1424, 2015.

7. John D Bernardin, Clinton J Stebbins, and Issam Mudawar. Mapping of impact and heat transfer regimes of water drops impinging on a polished surface. International Journal of Heat and Mass Transfer, 40(2):247-267, 1997.

8. YM Qiao and S Chandra. Experiments on adding a surfactant to water drops boiling on a hot surface. In Proceedings of the Royal Society of London A: Mathematical, Physical and Engineering Sciences, volume 453, pages 673-689. The Royal Society, 1997.

9. Tuan Tran, Hendrik JJ Staat, Andrea Prosperetti, Chao Sun, and Detlef Lohse. Drop impact on superheated surfaces. Physical Review Letters, 108(3):036101, 2012.

10. Tuan Tran, Hendrik JJ Staat, Arturo Susarrey-Arce, Tobias C Foertsch, Arie van Houselt, Han JGE Gardeniers, Andrea Prosperetti, Detlef Lohse, 
and Chao Sun. Droplet impact on superheated micro-structured surfaces. Soft Matter, 9(12):3272-3282, 2013.

11. Minori Shirota, Michiel AJ van Limbeek, Chao Sun, Andrea Prosperetti, and Detlef Lohse. Dynamic leidenfrost effect: relevant time and length scales. Physical Review Letters, 116(6):064501, 2016.

12. Hyungdae Kim, Bao Truong, Jacopo Buongiorno, and Lin-Wen Hu. On the effect of surface roughness height, wettability, and nanoporosity on leidenfrost phenomena. Applied Physics Letters, 98(8):083121, 2011.

13. Hrudya Nair, Hendrik JJ Staat, Tuan Tran, Arie van Houselt, Andrea Prosperetti, Detlef Lohse, and Chao Sun. The leidenfrost temperature increase for impacting droplets on carbon-nanofiber surfaces. Soft matter, 10(13):2102-2109, 2014.

14. Daniel Arnaldo del Cerro, Alvaro G Marin, Gertwillem RBE Roömer, B Pathiraj, Detlef Lohse, and Albertus J Huis in 't Veld. Leidenfrost point reduction on micropatterned metallic surfaces. Langmuir, 28(42):1510615110, 2012.

15. Cristian E Clavijo, Julie Crockett, and Daniel Maynes. Hydrodynamics of droplet impingement on hot surfaces of varying wettability. International Journal of Heat and Mass Transfer, 108:1714-1726, 2017.

16. Hyuk-min Kwon, James C Bird, and Kripa K Varanasi. Increasing leidenfrost point using micro-nano hierarchical surface structures. Applied Physics Letters, 103(20):201601, 2013.

17. D Toghraie Semiromi and AR Azimian. Molecular dynamics simulation of nonodroplets with the modified lennard-jones potential function. Heat and Mass Transfer, 47(5):579-588, 2011.

18. Jianguo Zhang, Frédéric Leroy, and Florian Müller-Plathe. Evaporation of nanodroplets on heated substrates: a molecular dynamics simulation study. Langmuir, 29(31):9770-9782, 2013.

19. Bo Shi and Vijay K Dhir. Molecular dynamics simulation of the contact angle of liquids on solid surfaces. The Journal of Chemical Physics, 130(3):034705, 2009.

20. Martin Horsch, Martina Heitzig, Calin Dan, Jens Harting, Hans Hasse, and Jadran Vrabec. Contact angle dependence on the fluid- wall dispersive energy. Langmuir, 26(13):10913-10917, 2010.

21. S Nishida, D Surblys, Y Yamaguchi, K Kuroda, M Kagawa, T Nakajima, and H Fujimura. Molecular dynamics analysis of multiphase interfaces based on in situ extraction of the pressure distribution of a liquid droplet on a solid surface. The Journal of Chemical Physics, 140(7):074707, 2014.

22. Hisao Yaguchi, Takeru Yano, and Shigeo Fujikawa. Molecular dynamics study of vapor-liquid equilibrium state of an argon nanodroplet and its vapor. Journal of Fluid Science and Technology, 5(2):180-191, 2010.

23. Pan Yi, D Poulikakos, J Walther, and George Yadigaroglu. Molecular dynamics simulation of vaporization of an ultra-thin liquid argon layer on a surface. International Journal of Heat and Mass Transfer, 45(10):2087$2100,2002$. 
24. Wenbin Zhang, Tongxu Yu, Jing Fan, Weijie Sun, and Zexian Cao. Droplet impact behavior on heated micro-patterned surfaces. Journal of Applied Physics, 119(11):114901, 2016.

25. Nahid Sedighi, Sohail Murad, and Suresh K Aggarwal. Molecular dynamics simulations of nanodroplet spreading on solid surfaces, effect of droplet size. Fluid Dynamics Research, 42(3):035501, 2010.

26. Kazumichi Kobayashi, Kazuki Konno, Hisao Yaguchi, Hiroyuki Fujii, Toshiyuki Sanada, and Masao Watanabe. Early stage of nanodroplet impact on solid wall. Physics of Fluids, 28(3):032002, 2016.

27. AKMM Morshed, Taitan C Paul, and Jamil A Khan. Effect of nanostructures on evaporation and explosive boiling of thin liquid films: a molecular dynamics study. Applied Physics A, 105(2):445-451, 2011.

28. Shiwei Zhang, Feng Hao, Haimu Chen, Wei Yuan, Yong Tang, and Xi Chen. Molecular dynamics simulation on explosive boiling of liquid argon film on copper nanochannels. Applied Thermal Engineering, 113:208214, 2017.

29. Gyoko Nagayama, Takaharu Tsuruta, and Ping Cheng. Molecular dynamics simulation on bubble formation in a nanochannel. International Journal of Heat and Mass Transfer, 49(23):4437-4443, 2006. 


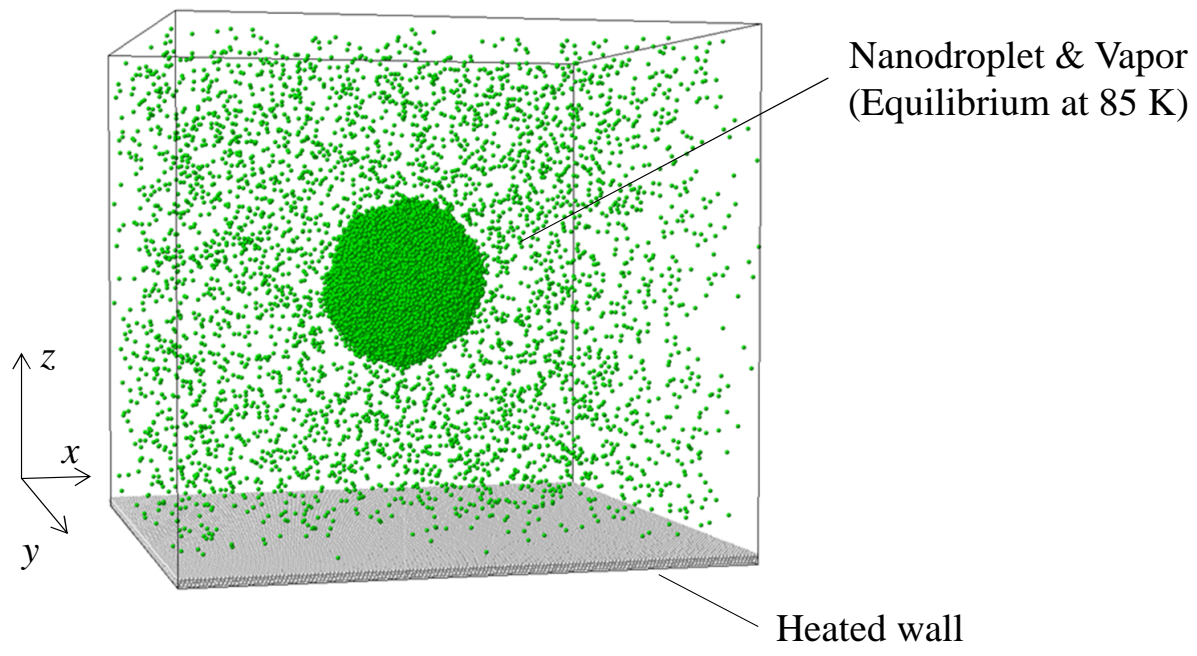

Fig. 1 Initial configration of the present MD study. 


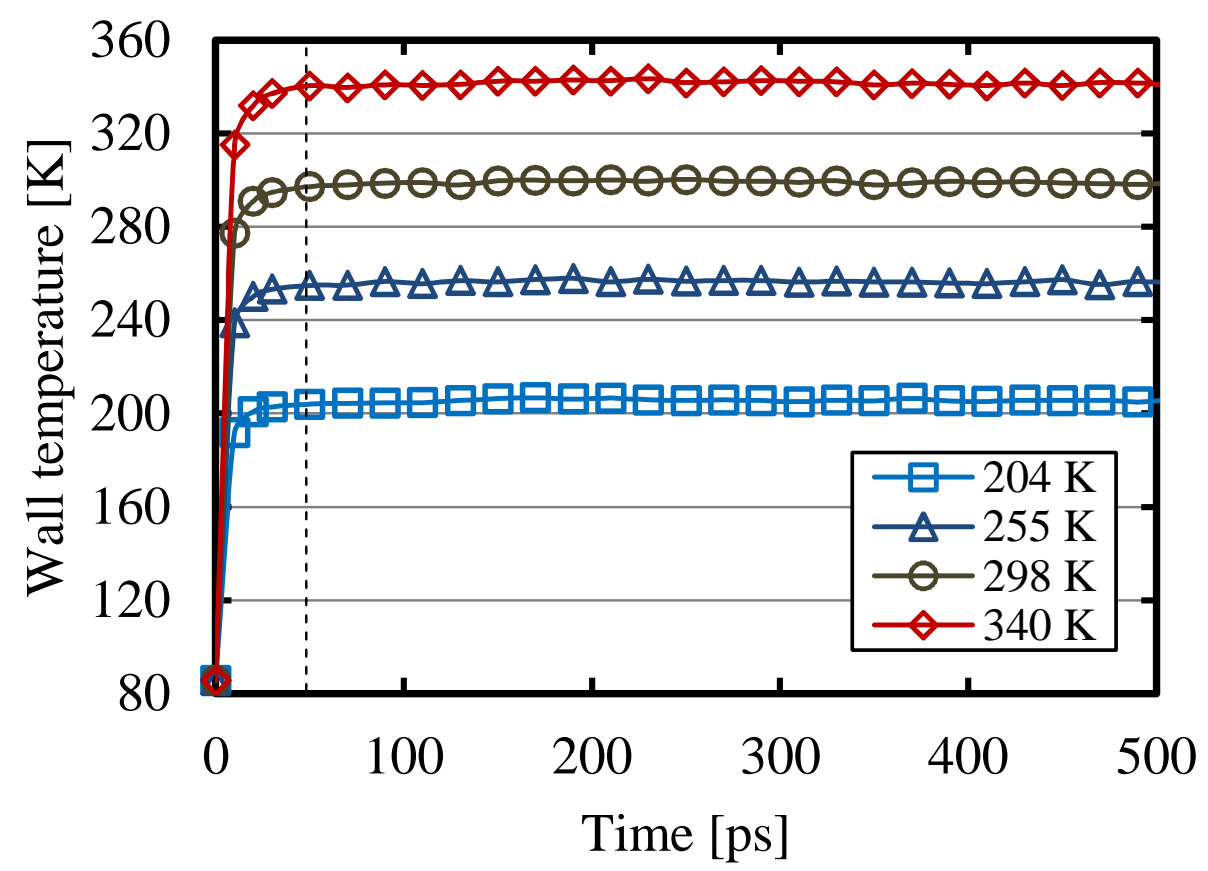

Fig. 2 Time evolutions of wall temperature. Dashed line represents the time liquid droplet impacted with $V_{0}=300 \mathrm{~m} / \mathrm{s}$. 
(a) $\alpha=0.07$

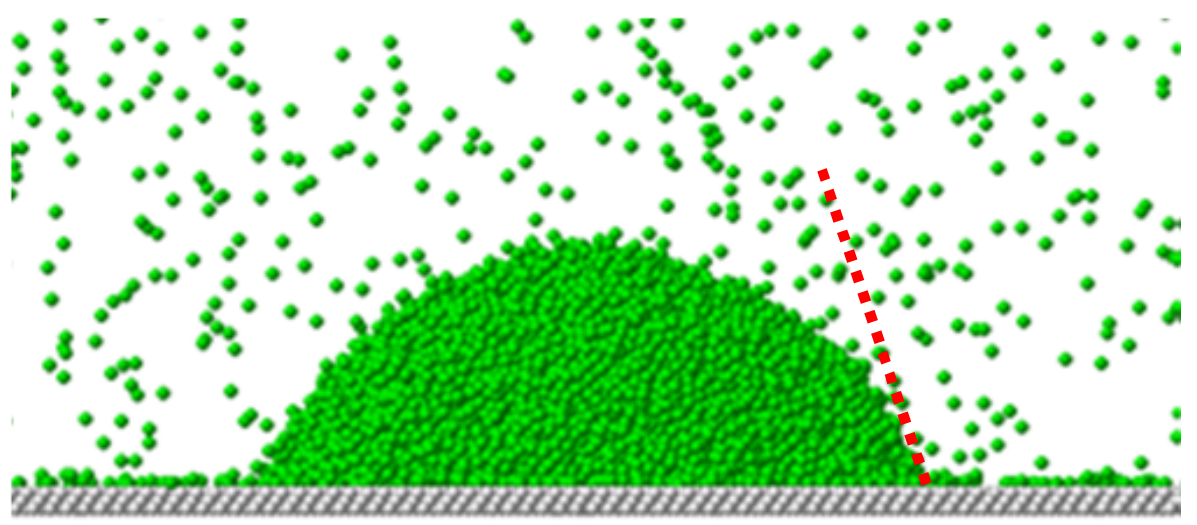

(b) $\alpha=1.0$

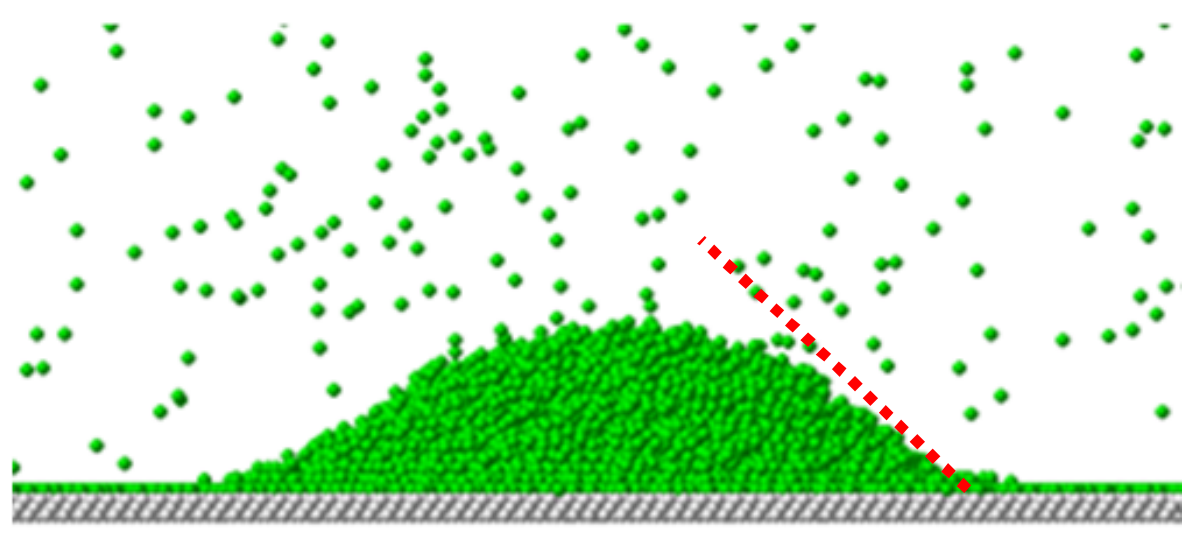

Fig. 3 Droplet shape after reaching equilibrium with $V_{0}=3 \mathrm{~m} / \mathrm{s}$ for (a) $\alpha=0.07$ and (b) $\alpha=1.0$. 


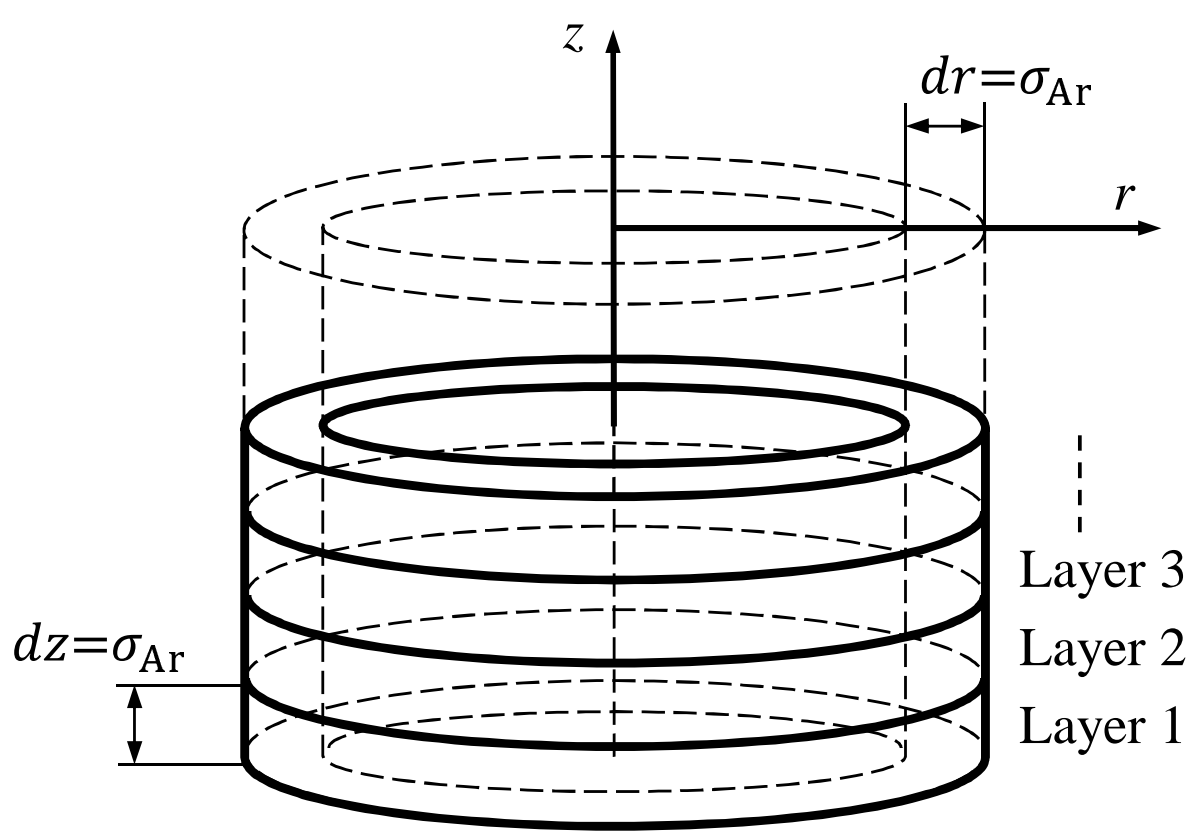

Fig. 4 Control volumes of the present MD study. 


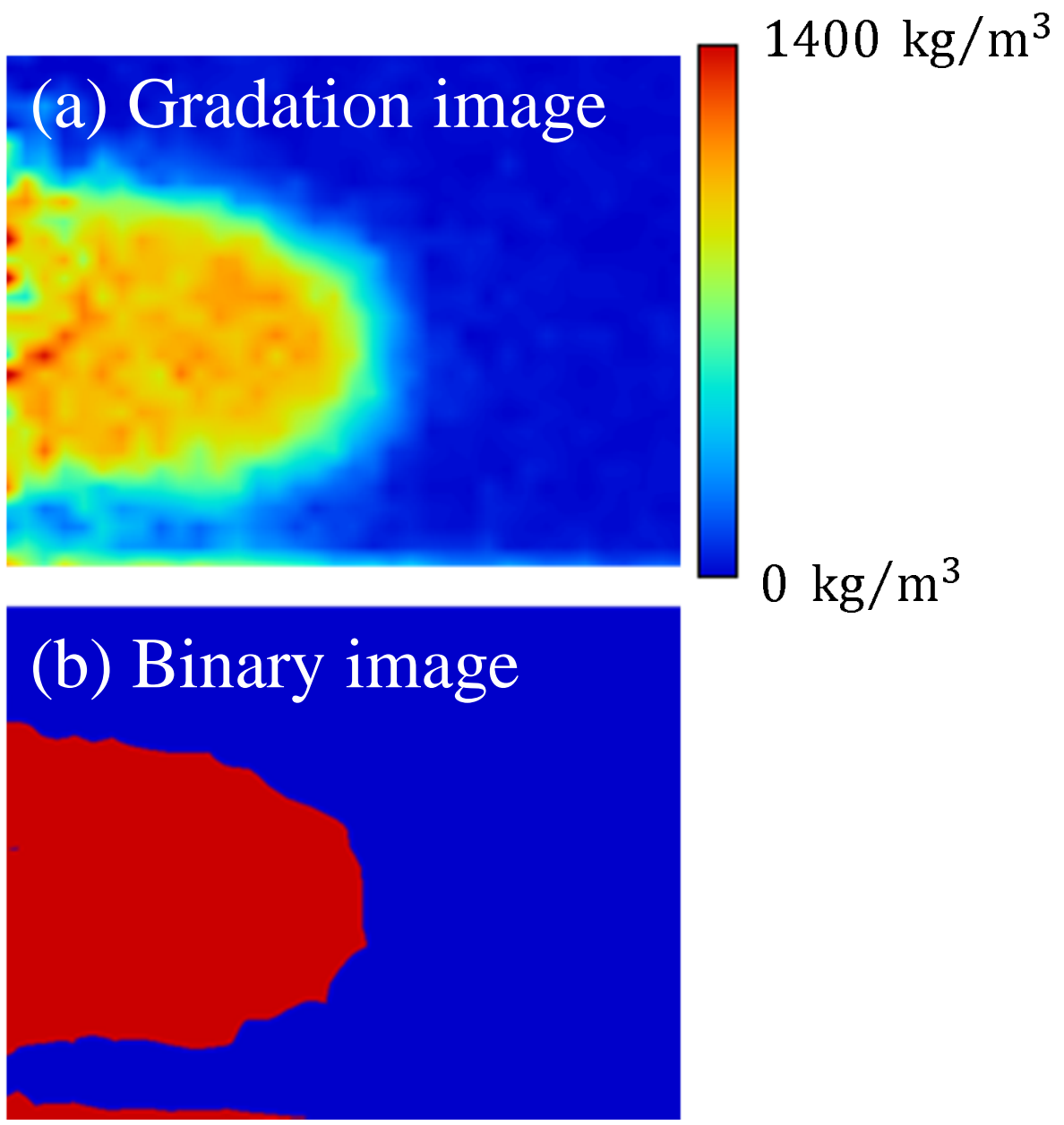

Fig. 5 Density fields of droplet with $V_{0}=100 \mathrm{~m} / \mathrm{s}$ for $\alpha=0.14$, (a) gradation image and (b) binary image. Wall temperature was $T_{w}=238 \mathrm{~K}$. 
(a) $\alpha=0.07$
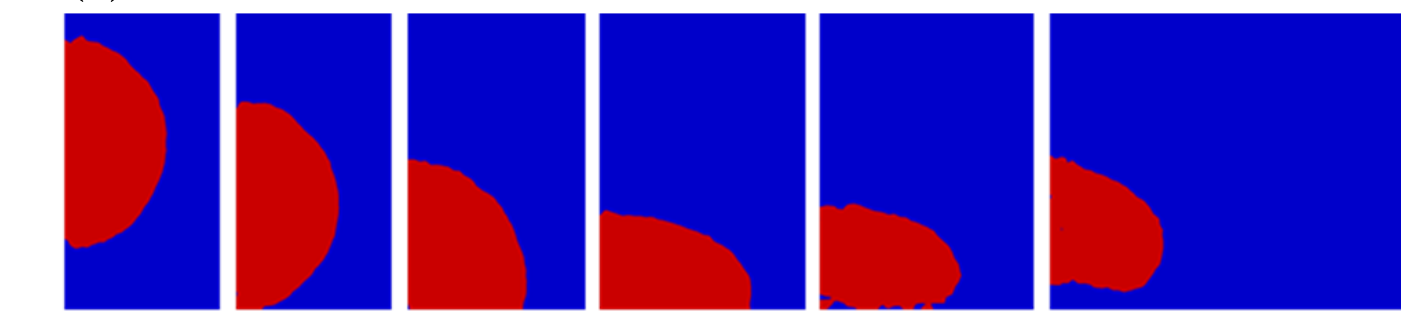

$t=-40 \mathrm{ps}$

0 ps

$40 \mathrm{ps}$

$100 \mathrm{ps}$

220 ps

$340 \mathrm{ps}$

(b) $\alpha=0.14$
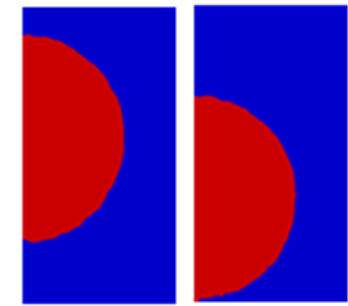

$t=-40 \mathrm{ps}$

0 ps

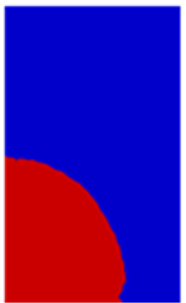

40 ps

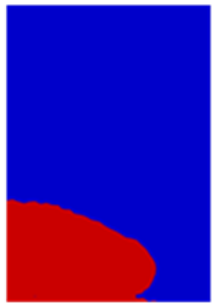

90 ps

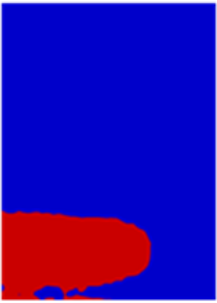

140 ps

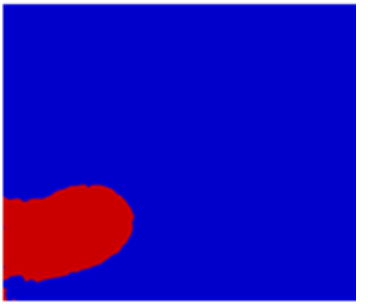

206 ps

(c) $\alpha=1.0$
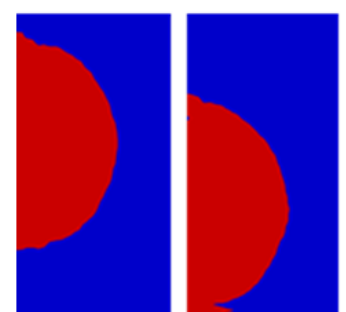

$t=-40 \mathrm{ps}$

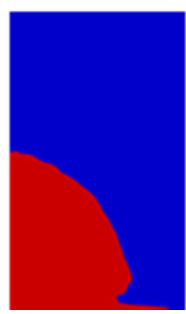

40 ps

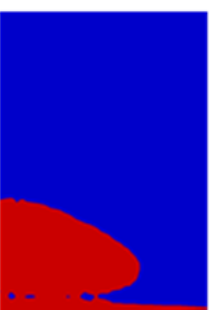

80 ps

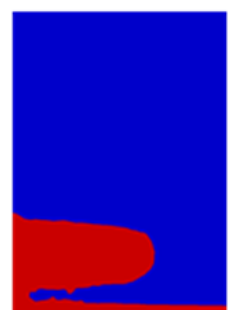

120 ps

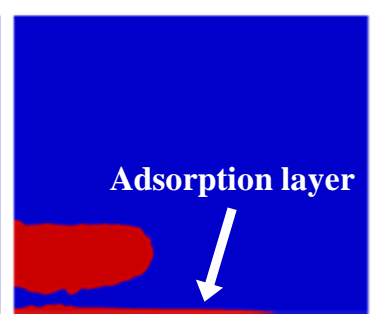

136 ps

Fig. 6 Droplet shapes until Leidenfrost effect occurred with $V_{0}=100 \mathrm{~m} / \mathrm{s}\left(T_{w}=340 \mathrm{~K}\right)$ for (a) $\alpha=0.07$, (b) $\alpha=0.14$, and (c) $\alpha=1.0$. The adsorption layer was formed on the solid surface for (c) $\alpha=1.0$. 


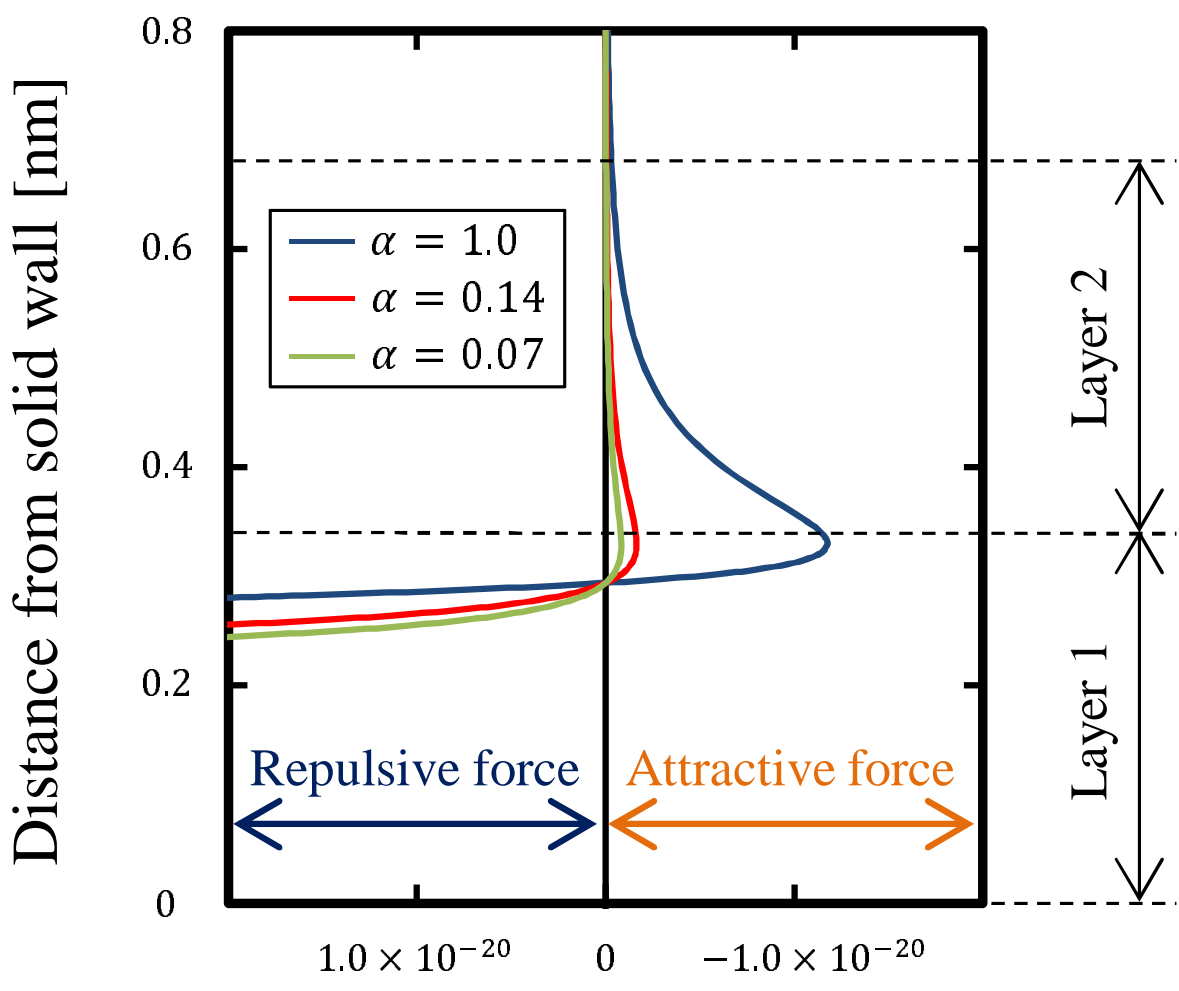

Intermolecular potential between liquid and solid molecules [J]

Fig. 7 Intermolecular potential between liquid and solid molecules for each value of $\alpha$. 

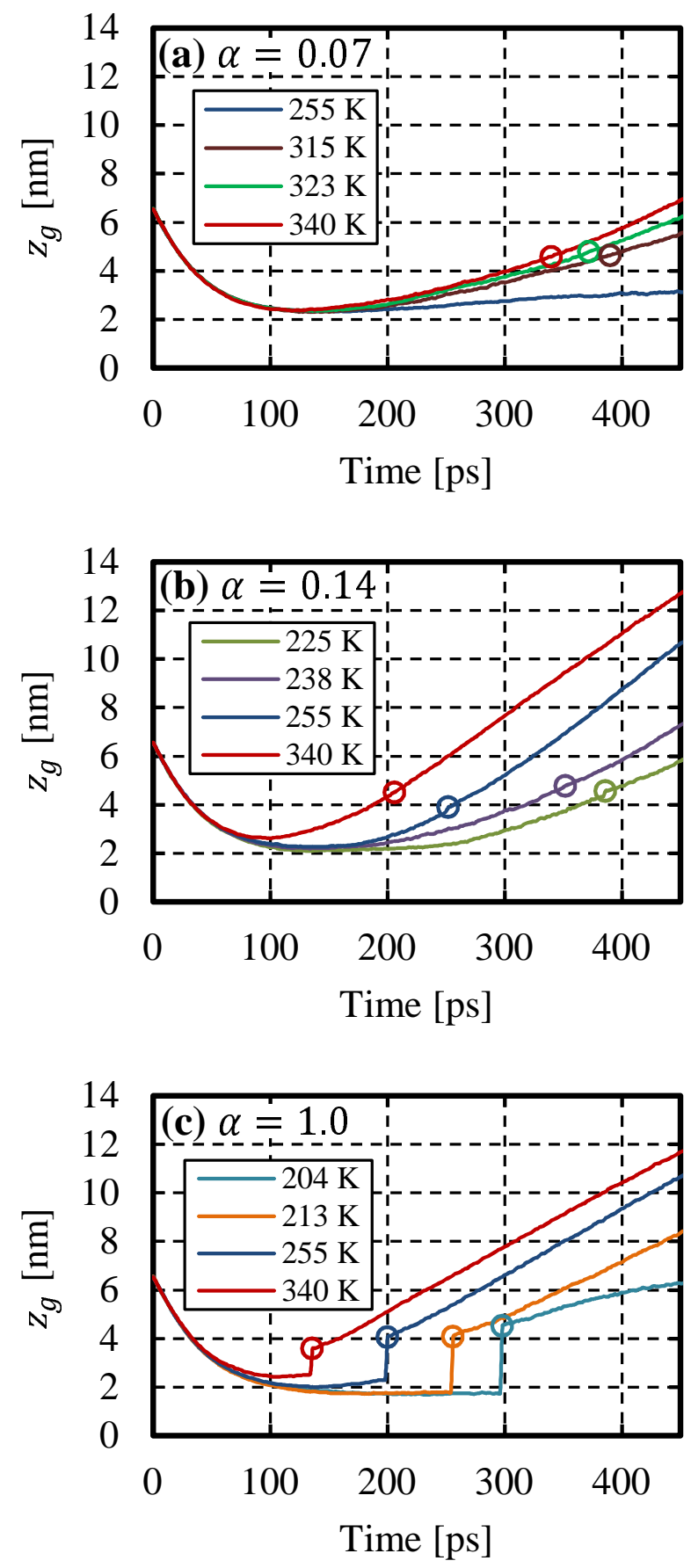

Fig. 8 Time evolutions of $z_{g}$ at $V_{0}=100 \mathrm{~m} / \mathrm{s}$ for (a) $\alpha=0.07$, (b) $\alpha=0.14$, and (c) $\alpha=1.0 . t=0 \mathrm{ps}$ was set at the time droplet impacted on solid wall. 

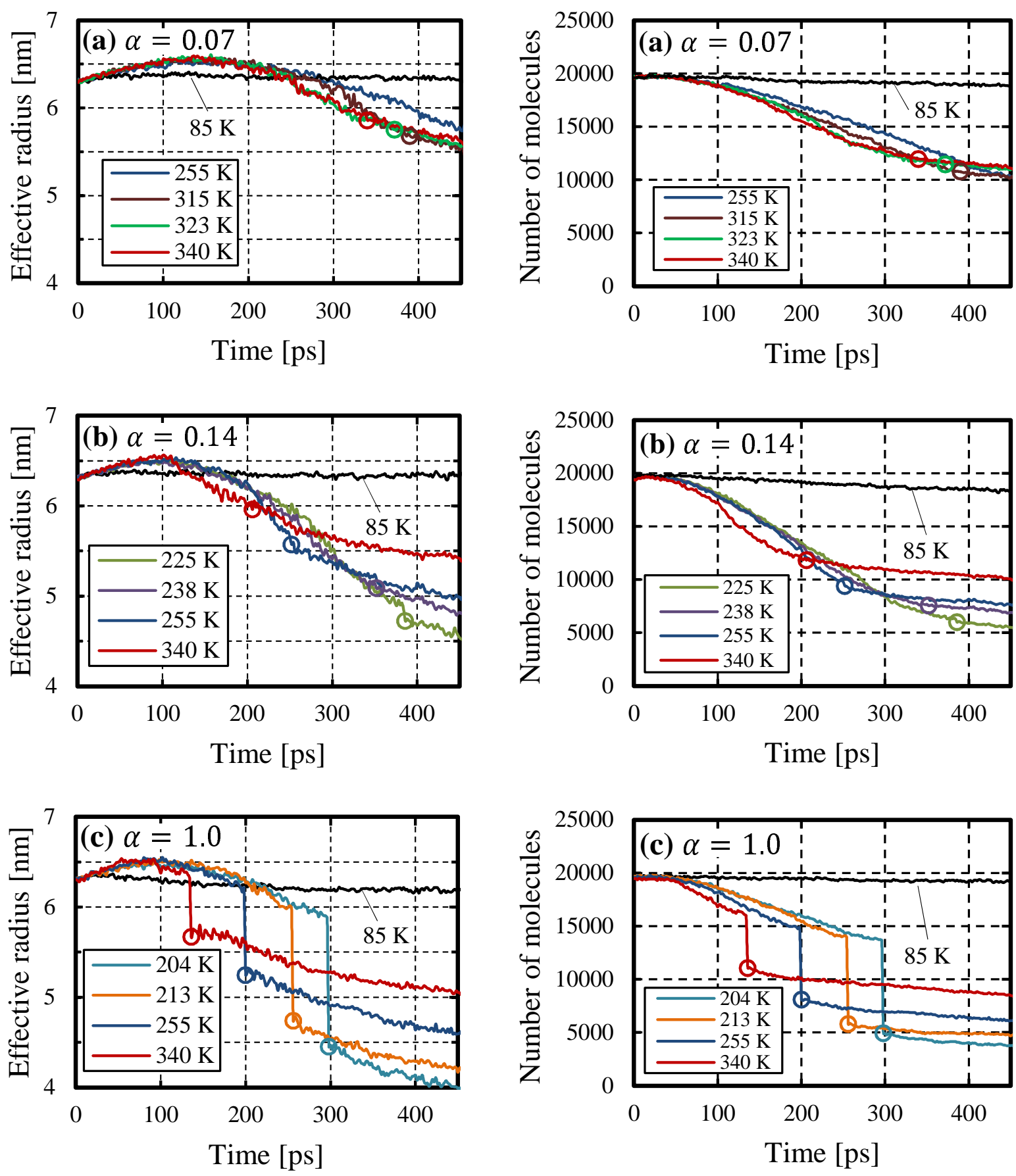

Fig. 9 Graphs in left column show time evolutions of effective radius of impacting droplet, and graphs in right column show the number of argon molecules in droplet with $V_{0}=100 \mathrm{~m} / \mathrm{s}$ for (a) $\alpha=0.07$, (b) $\alpha=0.14$, and (c) $\alpha=1.0 . t=0$ ps was set at the time droplet impacted on solid wall. 
(a) $\alpha=0.07$

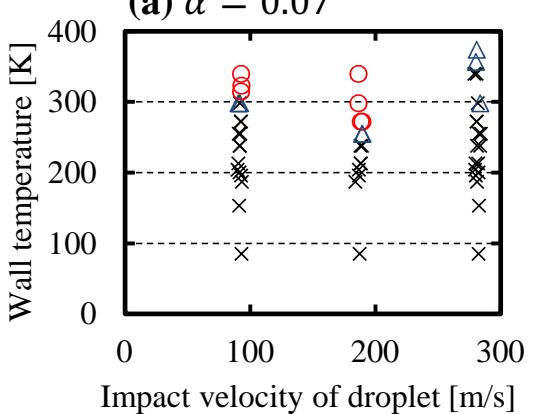

Impact velocity of droplet $[\mathrm{m} / \mathrm{s}]$ (b) $\alpha=0.14$

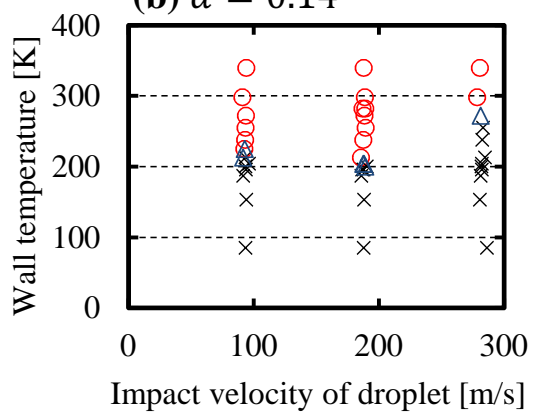

(c) $\alpha=1.0$

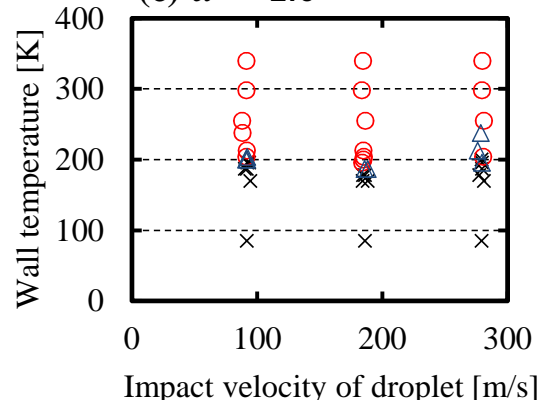

Fig. 10 Phase diagram of Leidenfrost effect for (a) $\alpha=0.07$, (b) $\alpha=0.14$, and (c) $\alpha=1.0$. Red circle represents case where Leidenfrost effect occurred, black cross represents case in which Leidenfrost effect did not occur, and blue triangle represents case where it is difficult to distinguish. 

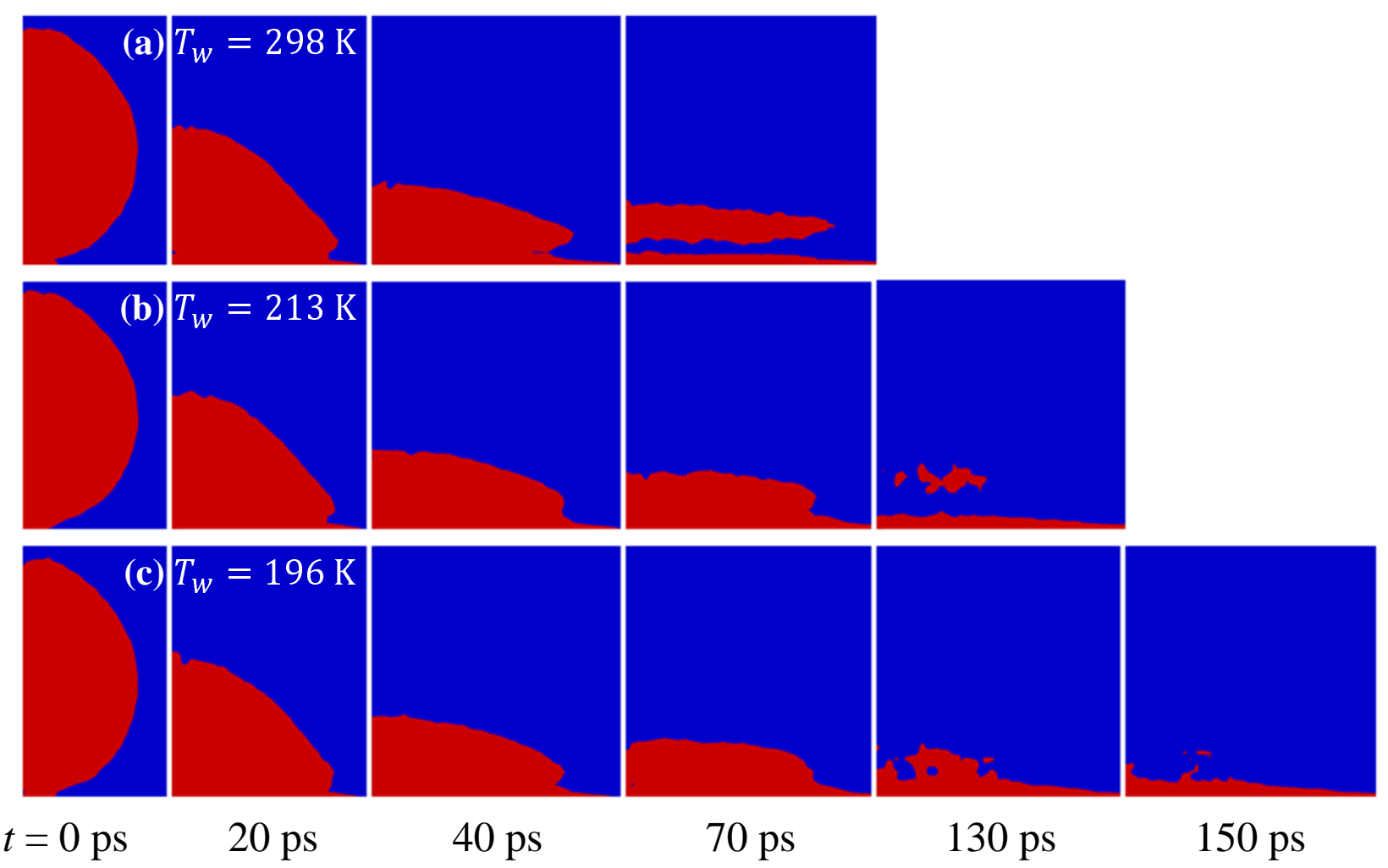

Fig. 11 Example of three patterns of droplet behavior after impacting for $\alpha=1.0$ and $V_{0}=300 \mathrm{~m} / \mathrm{s}$ : (a) Leidenfrost effect occurred, (b) difficult to distinguish, and (c) not occur. Wall temperature $T_{w}=$ (a) $298 \mathrm{~K}$, (b) $213 \mathrm{~K}$, and (c)196 K, respectively. $t=0$ ps was set at the time droplet impacted on solid wall. 
(a) $\alpha=0.07$

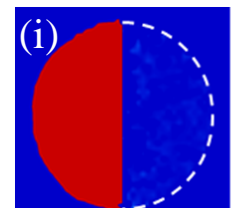

$t=0 \mathrm{ps}$

(b) $\alpha=0.14$

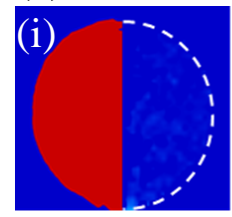

$t=0 \mathrm{ps}$

(c) $\alpha=1.0$

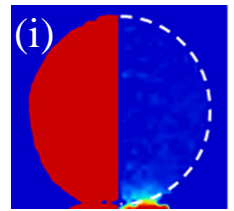

$t=0 \mathrm{ps}$

(a) $\alpha=0.07$

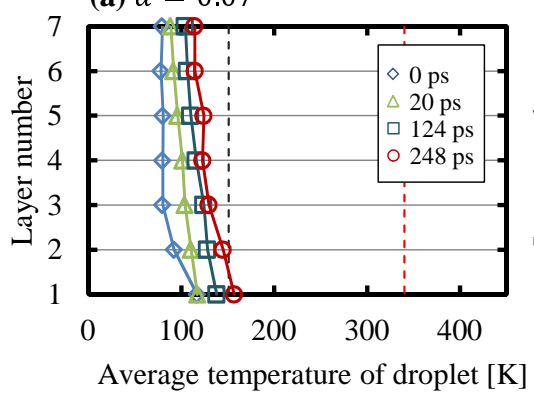

20 ps

$20 \mathrm{ps}$

20 ps
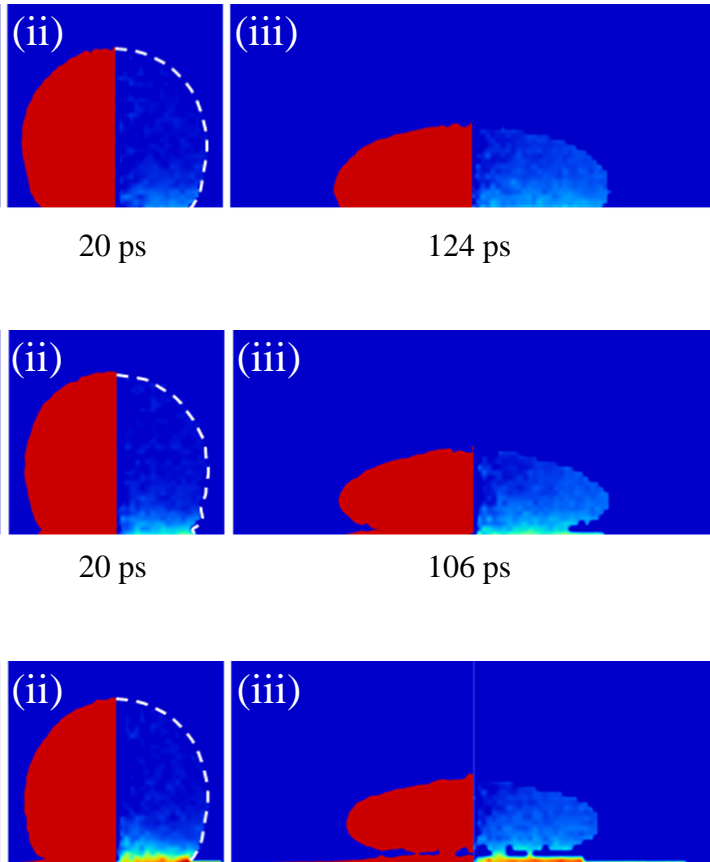

$106 \mathrm{ps}$

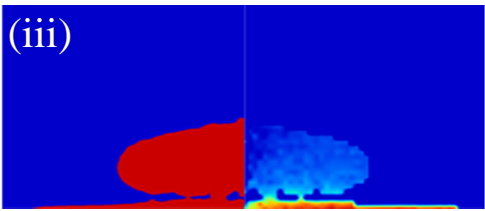

104 ps

(b) $\alpha=0.14$

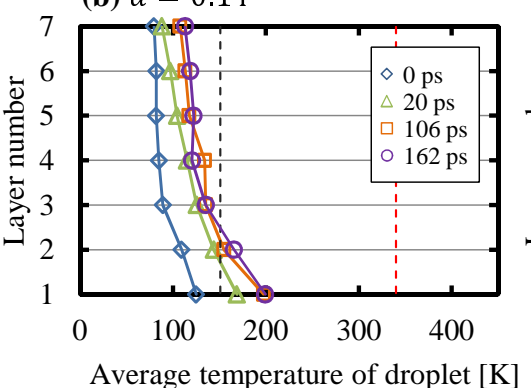

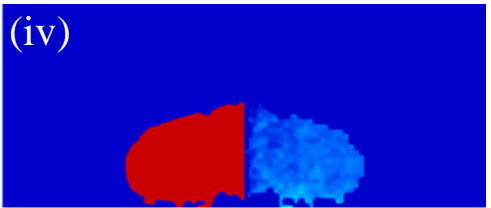

248 ps

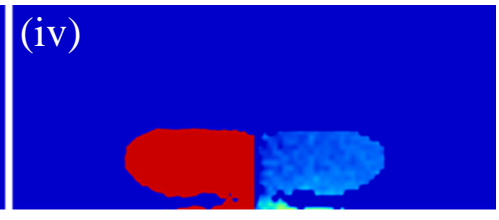

162 ps

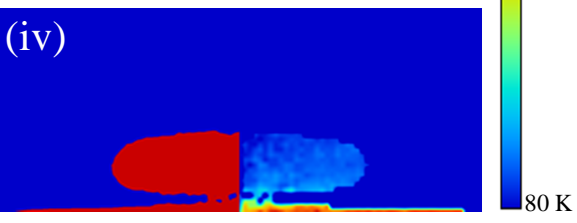

120 ps

(c) $\alpha=1.0$

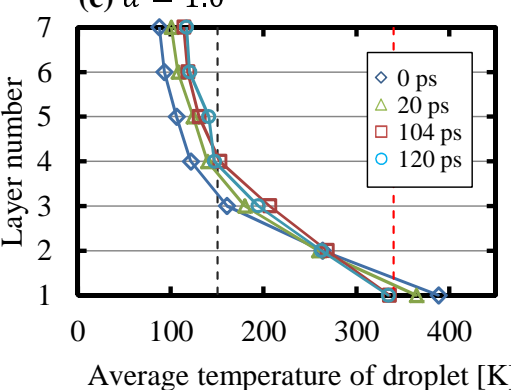

Fig. 12 Images in three rows show droplet shape (left part) and temperature field (right part) at $V_{0}=100 \mathrm{~m} / \mathrm{s}$ and $T_{w}=340 \mathrm{~K}$ for (a) $\alpha=0.07$, (b) $\alpha=0.14$, and (c) $\alpha=1.0$. $t=0$ ps was set at the time droplet impacted on solid wall. Graphs corresponding to upper images show average temperature in each layer of control volumes. Black dashed line represents critical temperature of argon $(150.687 \mathrm{~K})$, and red dashed line represents wall temperature $(340 \mathrm{~K})$. 